\title{
Six DOF Robotic Arm Prototype Modelling By Matlab
}

\author{
Alaa SAADAH, ${ }^{1}$ Géza HUSI ${ }^{2}$ \\ University of Debrecen, Mechatronic Engineering Department, Debrecen, Hungary \\ 1 alaa.saadah@eng.unideb.hu \\ ${ }^{2}$ husigeza@eng.unideb.hu
}

\begin{abstract}
The study in this paper allows us to control the manipulator and achieve any desired position and orientation. The Forward Kinematics was done using Denavait Hartenberg (DH) parameters, also the forward kinematics equations and homogenous transformation matrix was validated using MATLAB Toolbox. The modeling was carried out using the Peter Corke robotics toolbox.

Finally, the forward kinematic study and the robot arm's movement equations were compared with practical measurements to make sure it fulfilled the desired purpose and that it could point to the desired coordinates with a precision of $\pm 0.5 \mathrm{~cm}$.
\end{abstract}

Keywords: 6-DOF Industrial manipulator, Forward Kinematics, Peter Corke robotics toolbox.

\section{Összefoglalás}

A tanulmány ebben a cikkben lehetővé teszi számunkra, hogy vezéreljük a manipulátort, és elérjünk minden kívánt helyzetet és orientációt.

A forward kinematics Denavait Hartenberg (DH)-paraméterekkel készült, a forward kinematics-egyenleteket és a homogén transzformációs mátrixot is validálták a MATLAB Toolbox segítségével. A modellezés pedig Peter Corke Robotics toolbox által lett elvégezve.

Végül a forward kinematics-vizsgálat és a robotkar mozgásának egyenletei gyakorlati méréssel lettek kiegészítve, hogy megbizonyosodjunk arról, hogy teljesítik-e a kívánt célt, és \pm 0,5 cm-es pontossággal mutat a kívánt koordinátákra.

Kulcsszavak: 6-DOF Industrial manipulator, Forward Kinematics, Peter Corke robotics toolbox.

\section{Introduction}

A fundamental tool in robot kinematics is the kinematics equations of the kinematic chains that form the robot. These non-linear equations are used to map the joint parameters to the configuration of the robot system. Kinematics equations are also used in biomechanics of the skeleton and computer animation of articulated characters.

Forward kinematics uses the kinematic equations of a robot to compute the position of the end-effector from specified values for the joint parameters. In the twenty first century, there is a heavy customer demand for high quality and accurate products. A large number of today's industries have shifted their focus toward the installation of the robotic arm in their assembly line for faster production.
One of the most challenging problems of the robotic system is kinematics study, particularly the inverse kinematics which aims to find the joint angles for given robotic configurations.

The manipulator of an industrial robot consists of a series of joints and links, ABB IRB 4400 manipulator prototype deals with the study of different joints, links and other aspect of the manipulator's physical construction.

\section{Forward Kinematics}

The forward kinematics problem is concerned with the relationship between the individual joints of the robot manipulator and position and orientation of the tool or end-effector [1]. 


\subsection{Links and joints}

Stated more formally, the forward kinematics problem is to determine the position and orientation of the end-effector, given the values for the joint variables of the robot. The joint variables are the angles between the links in the case of prismatic or sliding [2].

Forward kinematics specifies the joint parameters and computes the configuration of the chain. For serial manipulators this is achieved by direct substitution of the joint parameters into the forward kinematics equations for the serial chain.

A wide variety of robot mechanisms can be described by categorizing their arrangement of joints and joint types. For the moment we will ignore the size and shape of links, and simply focus on broad categorization [3].

First, there are three typical joint types, each describing the form of relative transformations allowed between the two links to which it is attached:

- Revolute: the attached links rotate about a common axis;

- Prismatic: the attached links translate about a common axis;

-Spherical: the attached links rotate about a point.

A robot manipulator with $n$ joints will have $n+1$ links, since each joint connects two links. We number the joints from 1 to $n$ and we number the links from 0 to $n$ starting from the base.

By this convention, joint $i$ connects link $i-1$ to link $i$ moves. Therefore, link 0 - the first link- is fixed and does not move when the joint is actuated.

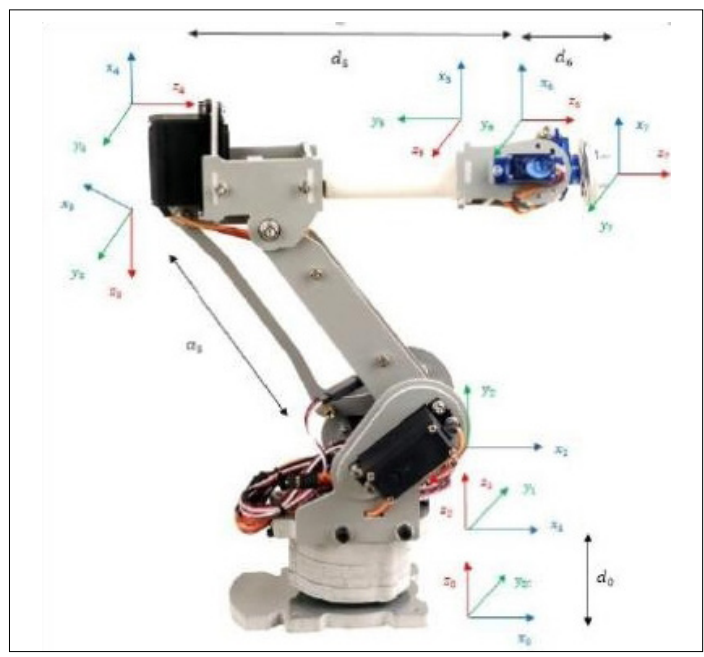

Figure 1. Robotic Arm Frame Assignment.

\subsection{Denavait Hartenberg}

We can get $T_{i}$ depend on four primary transformation:

$$
T_{i}=\operatorname{Rot}\left(z, \theta_{i}\right) \operatorname{Trans}\left(z, d_{i}\right) \operatorname{Trans}\left(x, a_{i}\right) \operatorname{Rot}\left(x, a_{i}\right)
$$

So, we can transfer from $i$ frame to $i+1$ frame.

$-\operatorname{Rot}\left(x, \alpha_{i}\right)$ : Rotation around $x$ axes $\alpha_{i}$ angle.

- Trans $\left(x, a_{i}\right)$ : Transformation $x$ axes $a_{i}$ angle.

$-\operatorname{Rot}\left(z, \theta_{i}\right)$ : Rotation around $z$ axes $\theta_{i}$ angle.

- Trans $\left(z, d_{i}\right)$ : Transformation $z$ axes $d_{i}$ angle.

$T_{i}$ final matrix will be the result of multiple of those matrixes . [3]

$$
\begin{aligned}
T_{i} & =\left[\begin{array}{llll}
c_{\theta i} & -s_{\theta i} & 0 & 0 \\
s_{\theta i} & c_{\theta i} & 0 & 0 \\
0 & 0 & 1 & 0 \\
0 & 0 & 0 & 1
\end{array}\right] \cdot\left[\begin{array}{cccc}
1 & 0 & 0 & 0 \\
0 & 1 & 0 & 0 \\
0 & 0 & 1 & d_{i} \\
0 & 0 & 0 & 1
\end{array}\right] \cdot\left[\begin{array}{cccc}
1 & 0 & 0 & a_{i} \\
0 & 1 & 0 & 0 \\
0 & 0 & 1 & 0 \\
0 & 0 & 0 & 1
\end{array}\right] \cdot\left[\begin{array}{cccc}
1 & 0 & 0 & 0 \\
0 & c_{\alpha i} & -s_{\alpha i} & 0 \\
0 & s_{\alpha i} & c_{\alpha i} & 0 \\
0 & 0 & 0 & 1
\end{array}\right] \\
T_{i} & =\left[\begin{array}{cccc}
c_{\theta i} & -s_{\theta i} c_{\alpha i} & s_{\theta i} s_{\alpha i} & a_{i} c_{\theta i} \\
s_{\theta i} & c_{\theta i} c_{\alpha i} & -c_{\theta i} s_{\alpha i} & a_{i} s_{\alpha i} \\
0 & s_{\alpha i} & c_{\alpha i} & d_{i} \\
0 & 0 & 0 & 1
\end{array}\right]
\end{aligned}
$$

$$
\alpha_{i}, \theta_{i}, d_{i}, a_{i}
$$

where,

- $a_{i}$ : the distance between $z_{i}$ to $z_{i+1}$ along the $x_{i}$ axis.

- $a_{i}$ : the angle between $z_{i}$ to $z_{i+1}$ között a $x_{i}$ axis.

- $d_{i}$ : the distance between $x_{i}$ to $x_{i+1}$ között a $z_{i}$ axis.

- $\theta_{i}$ : the angle between $x_{i}$ és $x_{i+1}$ között a $z_{i}$ axis.

\subsection{Robotic Arm DH Parameters}

Table 1. Denavait Hartenberg Parameters [5].

\begin{tabular}{|c|c|c|c|c|}
\hline $\boldsymbol{i}$ & $\boldsymbol{\theta}_{\boldsymbol{i}-\mathbf{1}}$ & $\boldsymbol{d}_{\boldsymbol{i}-\mathbf{1}}$ & $\boldsymbol{a}_{\boldsymbol{i}}$ & $\boldsymbol{a}_{\boldsymbol{i}}$ \\
\hline 1 & 0 & $d_{0}$ & 0 & 0 \\
\hline 2 & $q_{1}$ & 0 & 0 & $90^{\circ}$ \\
\hline 3 & $q_{2}$ & 0 & $a_{3}$ & 0 \\
\hline 4 & $90^{\circ}+q_{3}$ & 0 & 0 & $90^{\circ}$ \\
\hline 5 & $q_{4}$ & $d_{4}$ & 0 & $-90^{\circ}$ \\
\hline 6 & $q_{5}$ & 0 & 0 & $90^{\circ}$ \\
\hline 7 & $q 6$ & $d_{6}$ & 0 & 0 \\
\hline
\end{tabular}

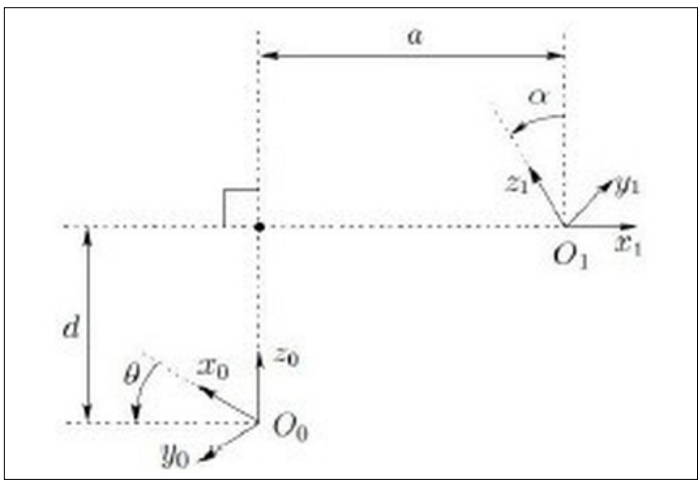

Figure 2. DH Frame Assignment [1] 


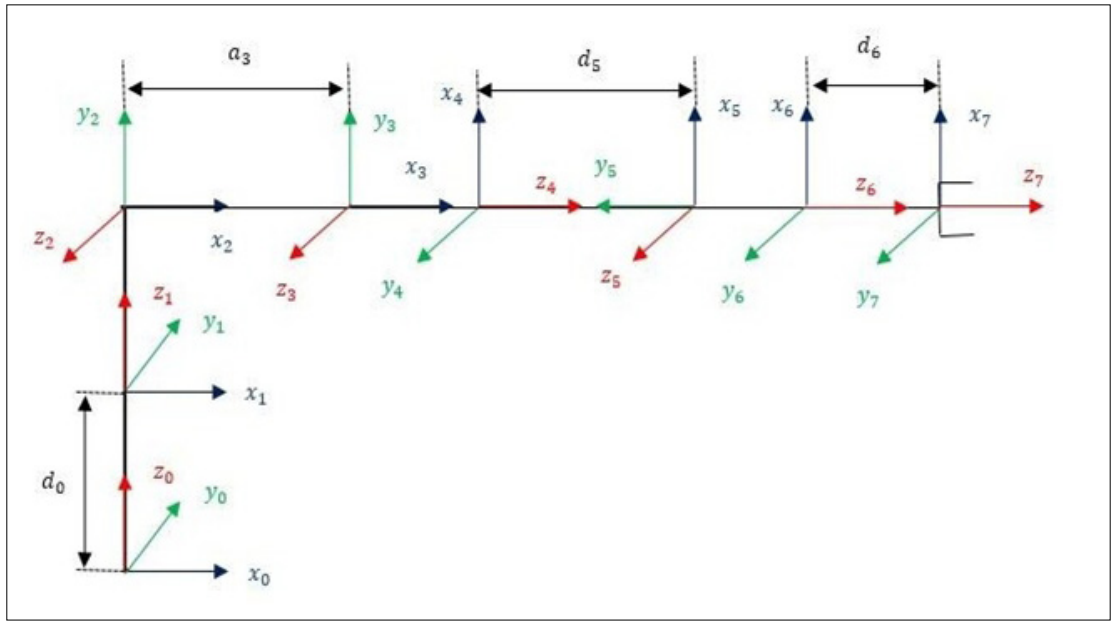

Figure 3. Coordinate Frames of the Robotic Arm.

$T_{1}^{0}=\left[\begin{array}{cccc}1 & 0 & 0 & 0 \\ 0 & 1 & 0 & 0 \\ 0 & 0 & 1 & d_{0} \\ 0 & 0 & 0 & 1\end{array}\right]$

(2)

$T_{2}^{1}=\left[\begin{array}{cccc}c_{q_{1}} & 0 & s_{q_{1}} & 0 \\ s_{q_{1}} & 0 & -c_{q_{1}} & 0 \\ 0 & 1 & 0 & 0 \\ 0 & 0 & 0 & 1\end{array}\right]$

$T_{3}^{2}=\left[\begin{array}{cccc}c_{q_{2}} & -s_{q_{2}} & 0 & c_{q_{2}} a_{3} \\ s_{q_{2}} & c_{q_{2}} & 0 & s_{q_{2}} a_{3} \\ 0 & 0 & 1 & 0 \\ 0 & 0 & 0 & 1\end{array}\right]$

$T_{4}^{3}=\left[\begin{array}{cccc}-s_{q_{3}} & 0 & c_{q_{3}} & 0 \\ c_{q_{3}} & 0 & s_{q_{3}} & 0 \\ 0 & 1 & 0 & 0 \\ 0 & 0 & 0 & 1\end{array}\right]$

$T_{5}^{4}=\left[\begin{array}{cccc}c_{q_{4}} & 0 & -s_{q_{4}} & 0 \\ s_{q_{4}} & 0 & c_{q_{4}} & 0 \\ 0 & -1 & 0 & d_{5} \\ 0 & 0 & 0 & 1\end{array}\right]$

$T_{6}^{5}=\left[\begin{array}{cccc}c_{q_{5}} & 0 & s_{q_{5}} & 0 \\ s_{q_{5}} & 0 & -c_{q_{5}} & 0 \\ 0 & 1 & 0 & 0 \\ 0 & 0 & 0 & 1\end{array}\right]$

$T_{7}^{6}=\left[\begin{array}{cccc}c_{q_{6}} & -s_{q_{6}} & 0 & 0 \\ s_{q_{6}} & c_{q_{6}} & 0 & 0 \\ 0 & 0 & 1 & d_{6} \\ 0 & 0 & 0 & 1\end{array}\right]$

$T_{7}^{0}=T_{1}^{0} \cdot T_{2}^{1} \cdot T_{3}^{2} \cdot T_{4}^{3} \cdot T_{5}^{4} \cdot T_{6}^{5} \cdot T_{7}^{6}=\left[\begin{array}{cccc}\mu_{x} & O_{x} & \alpha_{x} & p_{x} \\ \mu_{y} & O_{y} & \alpha_{y} & p_{y} \\ \mu_{z} & o_{z} & \alpha_{z} & p_{z} \\ 0 & 0 & 0 & 1\end{array}\right]$

$$
\begin{aligned}
\mu_{x}= & -c_{q_{1}} c_{q_{2}} s_{q_{3}} c_{q_{4}} c_{q_{5}} c_{q_{6}}+c_{q_{1}} c_{q_{2}} s_{q_{3}} s_{q_{4}} s_{q_{6}} \\
& -c_{q_{1}} c_{q_{2}} c_{q_{3}} s_{q_{5}} c_{q_{6}}-c_{q_{1}} s_{q_{2}} c_{q_{3}} c_{q_{4}} c_{q_{5}} c_{q_{6}} \\
& +c_{q_{1}} s_{q_{2}} c_{q_{3}} s_{q_{4}} s_{q_{6}}+c_{q_{1}} s_{q_{2}} s_{q_{3}} s_{q_{5}} c_{q_{6}} \\
& +s_{q_{1}} s_{q_{4}} c_{q_{5}} c_{q_{6}}+s_{q_{1}} c_{q_{4}} s_{q_{6}} \\
\mu_{y}= & -s_{q_{1}} c_{q_{2}} s_{q_{3}} c_{q_{4}} c_{q_{5}} c_{q_{6}}+s_{q_{1}} c_{q_{2}} s_{q_{3}} s_{q_{4}} s_{q_{6}} \\
& -s_{q_{1}} c_{q_{2}} c_{q_{3}} s_{q_{5}} c_{q_{6}}-s_{q_{1}} s_{q_{2}} c_{q_{3}} c_{q_{4}} c_{q_{5}} c_{q_{6}} \\
& +s_{q_{1}} s_{q_{2}} c_{q_{3}} s_{q_{4}} s_{q_{6}}+s_{q_{1}} s_{q_{2}} s_{q_{3}} s_{q_{5}} c_{q_{6}} \\
& -c_{q_{1}} s_{q_{4}} c_{q_{5}} c_{q_{6}}-c_{q_{1}} c_{q_{4}} s_{q_{6}}
\end{aligned}
$$

$$
\begin{aligned}
\mu_{z}= & -s_{q_{2}} s_{q_{3}} c_{q_{4}} c_{q_{5}} c_{q_{6}}+s_{q_{2}} s_{q_{3}} s_{q_{4}} s_{q_{6}} \\
& -s_{q_{2}} c_{q_{3}} s_{q_{5}} c_{q_{6}}+c_{q_{2}} c_{q_{3}} c_{q_{4}} c_{q_{5}} c_{q_{6}} \\
& -c_{q_{2}} c_{q_{3}} s_{q_{4}} s_{q_{6}}-c_{q_{2}} s_{q_{3}} s_{q_{5}} c_{q_{6}} \\
o_{x}= & c_{q_{1}} c_{q_{2}} s_{q_{3}} c_{q_{5}} s_{q_{6}}+c_{q_{1}} c_{q_{2}} s_{q_{3}} s_{q_{4}} s_{q_{6}} \\
& +c_{q_{1}} c_{q_{2}} c_{q_{3}} s_{q_{5}} s_{q_{6}}+c_{q_{1}} s_{q_{2}} c_{q_{3}} c_{q_{5}} s_{q_{6}} \\
& +c_{q_{1}} s_{q_{2}} c_{q_{3}} s_{q_{4}} s_{q_{6}}-s_{q_{1}} s_{q_{4}} c_{q_{5}} s_{q_{6}} \\
& +s_{q_{1}} c_{q_{4}} c_{q_{6}}
\end{aligned}
$$

$$
\begin{aligned}
O_{y}= & s_{q_{1}} c_{q_{2}} s_{q_{3}} c_{q_{5}} s_{q_{6}}+s_{q_{1}} c_{q_{2}} s_{q_{3}} s_{q_{4}} s_{q_{6}} \\
& +s_{q_{1}} c_{q_{2}} c_{q_{3}} s_{q_{5}} s_{q_{6}}+s_{q_{1}} s_{q_{2}} c_{q_{3}} c_{q_{5}} s_{q_{6}} \\
& +s_{q_{1}} s_{q_{2}} c_{q_{3}} s_{q_{4}} s_{q_{6}}-s_{q_{1}} s_{q_{2}} s_{q_{3}} s_{q_{5}} s_{q_{6}} \\
& +c_{q_{1}} s_{q_{4}} c_{q_{5}} s_{q_{6}}-c_{q_{1}} c_{q_{4}} c_{q_{6}}
\end{aligned}
$$

$$
\begin{aligned}
O_{z}= & s_{q_{2}} s_{q_{3}} c_{q_{5}} s_{q_{6}}+s_{q_{2}} s_{q_{3}} s_{q_{4}} s_{q_{6}} \\
& +s_{q_{2}} c_{q_{3}} s_{q_{5}} s_{q_{6}}-c_{q_{2}} c_{q_{3}} c_{q_{5}} s_{q_{6}} \\
& -c_{q_{2}} c_{q_{3}} s_{q_{4}} s_{q_{6}}+c_{q_{2}} s_{q_{3}} s_{q_{5}} s_{q_{6}}
\end{aligned}
$$

$$
\begin{aligned}
a_{x}= & -c_{q_{1}} c_{q_{2}} s_{q_{3}} c_{q_{4}} s_{q_{5}}+c_{q_{1}} c_{q_{2}} c_{q_{3}} c_{q_{5}} \\
& -c_{q_{1}} s_{q_{2}} c_{q_{3}} c_{q_{4}} s_{q_{5}}-c_{q_{1}} s_{q_{2}} s_{q_{3}} c_{q_{5}} \\
& +s_{q_{1}} s_{q_{4}} s_{q_{5}}
\end{aligned}
$$




$$
\begin{aligned}
a_{y}= & -s_{q_{1}} c_{q_{2}} s_{q_{3}} c_{q_{4}} s_{q_{5}}+s_{q_{1}} c_{q_{2}} c_{q_{3}} c_{q_{5}} \\
& -s_{q_{1}} s_{q_{2}} c_{q_{3}} c_{q_{4}} s_{q_{5}}-s_{q_{1}} s_{q_{2}} s_{q_{3}} c_{q_{5}} \\
& -c_{q_{1}} s_{q_{4}} s_{q_{5}} \\
a_{z}= & -s_{q_{2}} s_{q_{3}} c_{q_{4}} s_{q_{5}}+s_{q_{2}} c_{q_{3}} c_{q_{5}} \\
& +c_{q_{2}} c_{q_{3}} c_{q_{4}} s_{q_{5}}+c_{q_{2}} s_{q_{3}} c_{q_{5}} \\
p_{x}= & -c_{q_{1}} c_{q_{2}} s_{q_{3}} c_{q_{4}} s_{q_{5}} d_{6}+c_{q_{1}} c_{q_{2}} c_{q_{3}} c_{q_{5}} d_{6} \\
& +c_{q_{1}} c_{q_{2}} c_{q_{3}} d_{4}-c_{q_{1}} s_{q_{2}} c_{q_{3}} c_{q_{4}} s_{q_{5}} d_{6} \\
& -c_{q_{1}} s_{q_{2}} s_{q_{3}} c_{q_{5}} d_{6}-c_{q_{1}} s_{q_{2}} s_{q_{3}} d_{4}+c_{q_{1}} c_{q_{2}} a_{3} \\
& +s_{q_{1}} s_{q_{4}} s_{q_{5}} d_{6} \\
p_{y}= & -s_{q_{1}} c_{q_{2}} s_{q_{3}} c_{q_{4}} s_{q_{5}} d_{6}+s_{q_{1}} c_{q_{2}} c_{q_{3}} c_{q_{5}} d_{6} \\
& +s_{q_{1}} c_{q_{2}} c_{q_{3}} d_{4}-s_{q_{1}} s_{q_{2}} c_{q_{3}} c_{q_{4}} s_{q_{5}} d_{6} \\
& -s_{q_{1}} s_{q_{2}} s_{q_{3}} c_{q_{5}} d_{6}-s_{q_{1}} s_{q_{2}} s_{q_{3}} d_{4}+s_{q_{1}} c_{q_{2}} a_{3} \\
& -c_{q_{1}} s_{q_{4}} s_{q_{5}} d_{6} \\
p_{z}= & -s_{q_{2}} s_{q_{3}} c_{q_{4}} s_{q_{5}} d_{6}+s_{q_{2}} c_{q_{3}} c_{q_{5}} d_{6}+s_{q_{2}} c_{q_{3}} d_{4} \\
& +c_{q_{2}} c_{q_{3}} c_{q_{4}} s_{q_{5}} d_{6}+c_{q_{2}} s_{q_{3}} c_{q_{5}} d_{6}+c_{q_{2}} s_{q_{3}} d_{4} \\
& +s_{q_{2}} a_{3}+d_{0} \\
&
\end{aligned}
$$

\section{Validation of forward kinematic model}

The forward kinematics and homogeneous transformation matrix have been validated using Peter Corke robotics toolbox with MATLAB [6].

Equation values and visual plots, together with the end- effector position and orientation of the robot arm in MATLAB, gives clear insight into the robot arm movement [7].

Various angles were set as input and the results compared and plotted as shown as the following figures.

Table 2. various angles as input

\begin{tabular}{|c|c|c|c|c|c|c|c|}
\hline $\begin{array}{c}\text { Ang- } \\
\text { le }\end{array}$ & $\boldsymbol{q}_{\mathbf{1}}$ & $\boldsymbol{q}_{\mathbf{2}}$ & $\boldsymbol{q}_{\mathbf{3}}$ & $\boldsymbol{q}_{\mathbf{4}}$ & $\boldsymbol{q}_{\mathbf{5}}$ & $\boldsymbol{q}_{\mathbf{6}}$ & $\boldsymbol{q}_{\mathbf{7}}$ \\
\hline 1 & 0 & 0 & 0 & 0 & 0 & 0 & 0 \\
\hline 2 & 0 & 90 & -45 & 0 & 0 & 0 & 0 \\
\hline 3 & 0 & 45 & 45 & -90 & 45 & 45 & 45 \\
\hline 4 & 0 & 45 & 0 & -90 & 45 & 45 & 45 \\
\hline
\end{tabular}
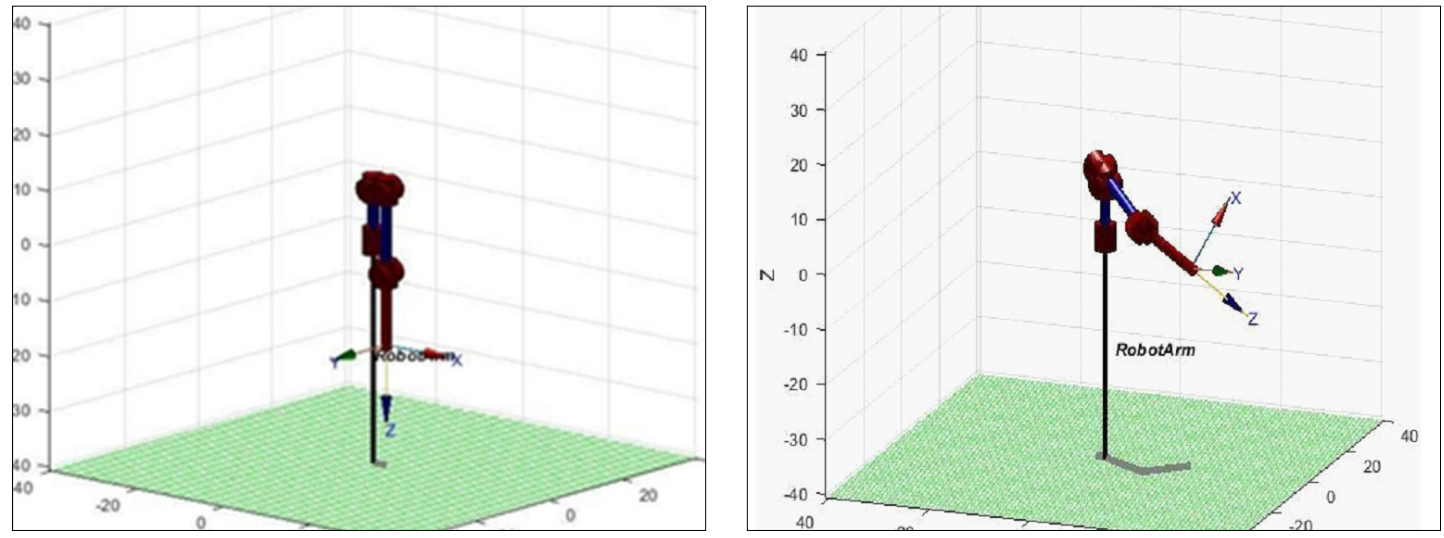

Figure 4. The plot for joint angle $1^{\text {st }}$ case in the table 2 .

Figure 6. The plot for joint angle $3^{\text {rd }}$ case in the table 2 .
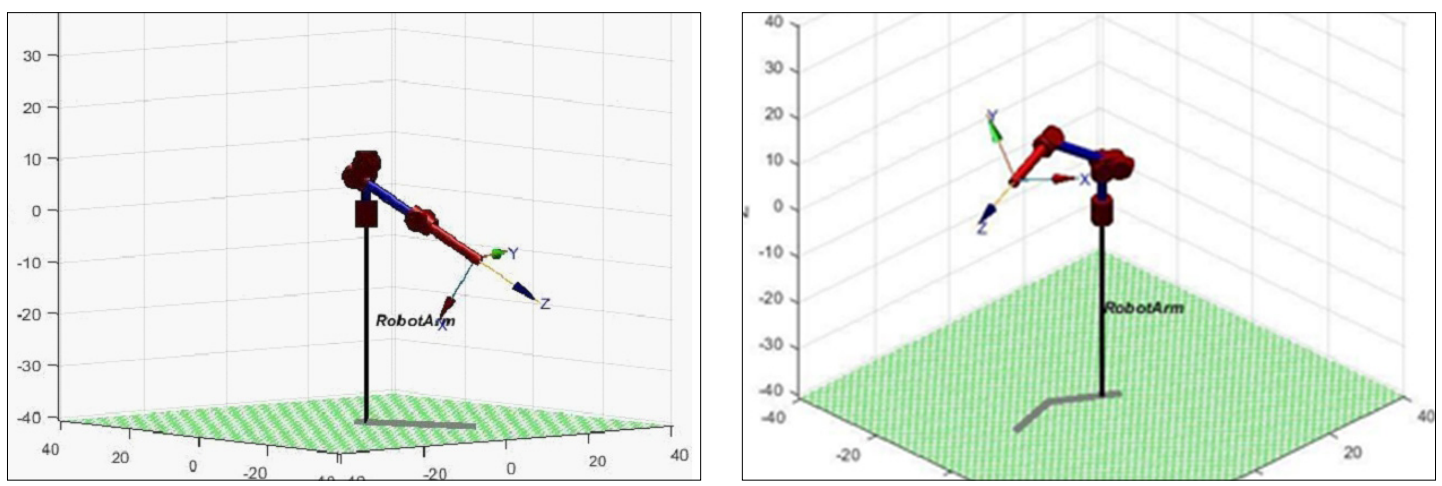

Figure 5. The plot for joint angle $2^{\text {nd }}$ case in the table 2 . 


\section{Conclusion}

The forward Kinematics model has been validated using MATLAB. The results from the forward kinematics model match the results from the homogeneous transformation matrix script result. [9] The positions obtained from the model have been compared with the actual performance of the robot to detect the end- effector position and orientation.

The robot achieves position precision within $\pm 0.5 \mathrm{~cm}$, this small deviation is due to many reasons e.g. mechanical coupling of the joints and non-linearity in mapping angles to low-level encoder ticks.

\section{References}

[1] Shrivastava S.: Forward Kinematics of Articulated Robotic Arm. International Journal of Research and Scientific Innovation, IV/VIII. (2017) 78-82.

[2] Iqbal J., Islam R., Khan H.: Modeling and Analysis of a 6 DOF Robotic Arm Manipulator. Canadian Journal on Electrical and Electronics Engineering, 3/6. (2012) 300-306.
[3] Zhang D., Wei B.: A Review on Model Reference Adaptive Control of Robotic Manipulators. Annual Reviews in Control, 43. (2017) 188-198.

[4] Hou Z.: Kinematics Analysis and Self-Collision Detection of Truss Type Multi-Robot. In: $52^{\text {nd }}$ CIRP Conference on Manufacturing Systems, Shanghai, China, 2019.

[5] Saadah A., Husi G.: Computing The Kinematics Study of a 6 DOF Industrial Manipulator Prototype By Matlab. Recent Innovations in Mechatronics, 7/1. (2020) 1-5. https://doi.org/10.17667/riim.2020.1/8.

[6] Corke P.: Robotics, Vision and Control. Fundamental Algorithms In MATLAB® Second, Completely Revised, Extended And Updated Edition. Springer, 2017.

[7] Krishnan M. G.: Kinematic Analysis and Validation of an Industrial. In: IEEE TENCON Conference, Kerala, 2019.

[8] Liu F.: Kinematic Analysis and Simulation of a 3-DOF robotic. In: IEEE International Conference on Computational Intelligence and Communication Technology, Ghaziabad, 2017.

[9] MATLAB 2018a, The MathWorks, Inc., Natick, Massachusetts, United States. 